\title{
HYDROMAGNETIC DYNAMO IN ASTROPHYSICAL JETS
}

\author{
A. SHUKUROV \\ Computing Center, Moscow University, Moscow 119899, Russia \\ and \\ D.D. SOKOLOFF \\ Physics Department, Moscow University, Moscow 119899, Russia
}

\begin{abstract}
The origin of a regular magnetic field in astrophysical jets is discussed. It is shown that jet plasma flow can generate a magnetic field provided the streamlines are helical. The dynamo of this type, known as the screw dynamo, generates magnetic fields with the dominant azimuthal wave number $m=1$ whose field lines also have a helical shape. The field concentrates into a relatively thin cylindrical shell and its configuration is favorable for the collimation and confinement of the jet plasma.
\end{abstract}

Key words: The screw dynamo - Astrophysical jets

Long, thin, well-collimated jets are a widespread companion of energetic activity in radio galaxies and young stars (see, e.g., the reviews of Begelman et al., 1984; Lada, 1985), and even in the Galactic center region (Mirabel et al., 1992). Polarization observations of the synchrotron emission of extragalactic jets indicate the presence of magnetic fields of a few $\mu \mathrm{G}$ strength at the kiloparsec scale. A high polarization of the synchrotron emission indicates a rather high degree of ordering of the magnetic field, although purely random two-dimensional magnetic field configurations can also produce polarized emission (Laing, 1981). It seems only reasonable to conclude that magnetic fields in the jets contain both regular and random components, as do the fields in all other astronomical objects. In many cases, polarization observations are compatible with a helical shape for the field lines of the ordered field.

In contrast to the rather detailed knowledge of the magnetic field morphology in extragalactic jets, very little is known about the plasma velocity patterns. This is due to the fact that extragalactic jets emit mainly a continuous, synchrotron emission, so that the classical method of the observational study of velocity fields based on the Doppler shifts of spectral lines does not work. As a result, our knowledge of the jet velocity fields is only rudimentary. However, there is an indirect evidence of a helical shape for the streamlines in the jets of quasars and BL Lac objects as discussed by Camenzind and Krockenberger (1992). The helical shape of both the streamlines and the magnetic lines is also a property of many self-similar MHD jet models.

The observational situation with stellar jets is quite the opposite. They contain rather dense thermal plasma, so that their velocity fields can be studied, at least in principle, by conventional methods of astronomy. Up to now, observational attempts have focused on the longitudinal velocity in stellar jets but, undoubtedly, information on the transverse velocity components and the velocity profiles across the jet will follow. However, up to now there is hardly any information about magnetic fields in stellar bipolar outflows, even though magnetic field is believed to play an important role in the formation, collimation and confinement of both extragalactic and stellar jets (see Begelman et al., 1984; Camenzind, 1990). As long as we might believe that the mechanisms of acceleration and collimation of the jet 
flows in extragalactic and stellar environments are physically similar, the observational information on these two types of astrophysical jets can be considered as complimentary, with the extragalactic jets giving clues to the typical or admissible magnetic field properties and the stellar jets providing the guidelines for the velocity field modelling.

Jet outflows originate in the vicinity of a compact object, e.g., a black hole or a compact stellar object surrounded by an accretion disk. Since accretion disks and close vicinities of black holes are a favorable site for the amplification of magnetic fields (see., e.g., Pudritz, 1981; Asseo, 1987; Camenzind, 1990; Stepinsky and Levy, 1991), the plasma in the jet inevitably carries magnetic field. If the field were frozen into the plasma, its longitudinal and transverse components would scale with the jet radius $R$ as $B_{\|} \propto R^{-2}$ and $B_{\perp} \propto R^{-1}$, respectively (Blandford and Rees, 1974). Near a black hole of mass $10^{8} M_{\odot}$, the inner radius of the accretion disk is about $10^{14} \mathrm{~cm}$ and the magnetic field strength can reach $10^{4} \mathrm{G}$. Then at a distance of $10 \mathrm{kpc}$ along the jet the longitudinal component of the frozen-in magnetic field would decay down to $10^{-11} \mathrm{G}$ for the jet opening angle of $0.1 \mathrm{rad}$. Meanwhile, equipartition estimates indicate that the longitudinal field decreases along the jet axis much more slowly than predicted by the flux freezing. For instance, in 3C 120 magnetic field is aligned with the jet axis and $B_{\mathrm{eq}} \simeq 16 \mathrm{mG}(R / 1 \mathrm{pc})^{-1}$ at distances from the center ranging between $\simeq 1 \mathrm{pc}$ and $\simeq 100 \mathrm{kpc}$ (Walker et al., 1987). Thus, the magnetic field cannot be frozen into the jet plasma (see, e.g., De Young, 1980; Perley, 1987; Königl, 1987) but it is amplified within the jet.

Such amplification can occur due to the conversion of the kinetic energy of electrically conducting, neutral plasma into magnetic energy. In general terms, such a process is called the hydromagnetic dynamo. One of the best-studied types of dynamo is the so-called mean-field dynamo, which is the subject of many contributions in this volume. This is a mechanism of magnetic field amplification in a turbulent flow with broken mirror symmetry (e.g., a helical random flow). Meanfield dynamos have been applied to extragalactic jets by De Young (1980) and Gvaramadze et al. (1988). However, it seems difficult to explain how turbulent motions in the jet acquire a sufficiently strong mean helicity in spite of the fact that the motions in the accretion disk, where the matter probably originates, are helical.

A smooth mean velocity field in the form of a jet, if having helical streamlines, can act as a dynamo without any mean helicity (known as the Ponomarenko dynamo, or the screw dynamo; we note, however, that in this dynamo the regular flow is helical itself). An axisymmetric flow with helical streamlines can amplify magnetic field provided the magnetic Reynolds number exceeds a critical value in the range between about 10 and $10^{2}$ depending on the distribution of the flow velocity along the jet radius (Lortz, 1968; Ponomarenko, 1973; Ruzmaikin et al., 1988; see also Lupyan and Shukurov, 1992). The screw dynamo belongs to the class of slow dynamos, which means that the growth time (defined as the reciprocal growth rate $\lambda$ ) of the magnetic field tends to infinity when the magnetic Reynolds number $R_{\mathrm{m}}$ increases. When the velocity profile along the cylindrical radius $r$ is smooth, we have $\lambda \propto R_{\mathrm{m}}^{-1 / 2}$ (Ruzmaikin et al., 1988) for small $m ; \lambda \propto R_{\mathrm{m}}^{-1 / 3}$ for $m \propto R_{\mathrm{m}}^{1 / 3}$ (Gilbert,1988).

Undoubtedly, $R_{\mathrm{m}}$ based on plasma conductivity is enormous in extragalactic 
jets, so that the growth time due to the screw dynamo might be expected to be improperly large. However, we recall that the magnetic field in extragalactic jets contains a relatively strong random component. It is only natural to assume that the plasma motions also are not purely regular but rather contain a random component as well. In a random flow, the turbulent magnetic Reynolds number is more relevant for the screw dynamo. The mixing-length expression for the turbulent magnetic diffusivity is $\nu \simeq v l / 3$, where $v$ is the turbulent velocity, which we can estimate to be of the order of the sound speed $c_{s}$, and $l$ is the turbulent scale, which we tentatively estimate as $l \simeq 0.1 R$ with $R$ the jet radius. Thus, $R_{\mathrm{m}}=V R / \nu \simeq 30 M$, where $V$ is the typical regular velocity and $M$ is the Mach number (Ruzmaikin et al., 1988). More precisely, here $V$ is that part of the total velocity which is involved in the magnetic field amplification, i.e., the shearing velocity represented by differential rotation and the radial gradient of the longitudinal velocity. For $M=O(1)$, the turbulent magnetic Reynolds number is expected to be in the range most favorable for an efficient action of the screw dynamo: on the one hand, it is high enough to exceed the generation threshold, on the other hand, $R_{\mathrm{m}}$ is only moderate, so that the growth time of the magnetic field can be short enough.

The dynamo action associated with the helical flow of the type considered here can be easily simulated numerically. The reason why the dynamo amplification of the magnetic field due to the screw dynamo has not been revealed in numerous MHD simulations of extragalactic jets, is the fact that the numerical models are based typically on ideal MHD codes. Meanwhile, the screw dynamo is impossible in an ideal fluid because the coupling of the azimuthal and axial magnetic field components in the induction equation, with the velocity field considered, occurs due to the magnetic diffusion term (this is why the screw dynamo belongs to the class of slow dynamos). Moreover, the magnetic field generated by the screw dynamo can be only nonaxisymmetric because the flow is axisymmetric (Cowling's theorem). Thus, only a three-dimensional dissipative model is suitable for a study of the dynamo.

It can be shown that the mode with the azimuthal wave number $m=1$ have the minimal critical $R m$ and probably is dominant in the dynamo-generated magnetic field, at least at the kinematic stage (see Sokoloff et al., 1989). The field lines represent helices whose pitch is related to the flow parameters (Ruzmaikin et al., 1988):

$$
\frac{B_{\phi}}{B_{z}}=\frac{k R}{m}=-\frac{R \omega^{\prime}\left(r_{0}\right)}{v_{z}^{\prime}\left(r_{0}\right)}
$$

where $k$ and $m$ are the longitudinal and azimuthal wave numbers of magnetic modes, respectively, the velocity field is represented as $\mathbf{V}=\left(0, r \omega(r), v_{z}(r)\right)$, with $(r, \phi, z)$ cylindrical coordinates, $r_{0}$ is the radius at which the magnetic field concentrates (under normal conditions, this radius is determined by the dynamo eigenfunction having the largest growth rate-cf. Reshetnyak et al., 1991), and prime denotes a derivative with respect to $r$. The typical maximum growth rate of the magnetic field is of the order of $10^{-2} V / R$ (Lupyan and Shukurov, 1992). The magnetic field reaches a maximum at some distance from the jet axis, and for $R_{\mathrm{m}} \gg 1$ it concentrates in a thin cylindrical shell of thickness $O\left(R_{\mathrm{m}}^{-1 / 4}\right)$ (Ruzmaikin et al., 1988). Although the latter estimate has been obtained for $R_{\mathrm{m}} \gg 1$, its accuracy is satisfactory even for 
moderate values of $R_{\mathrm{m}}$ (Ruzmaikin et al., 1989). The above estimates have been obtained for the kinematic dynamo, i.e., the stage of exponential time growth of the magnetic field. The field growth saturates plausibly when the magnetic energy density becomes comparable to the kinetic energy density of the motions responsible for the dynamo action. Since the field is generated due to the velocity shear, we can propose the following heuristic estimate of the steady-state field strength:

$$
B \simeq(4 \pi \rho)^{1 / 2} V_{\text {shear }},
$$

where $\rho$ is the plasma density and $V_{\text {shear }}$ is the shearing velocity, so that the steadystate field strength can be reasonable even for very large total velocities in the jet provided the velocity shear is relatively small. As the dynamo-generated magnetic field has a strong azimuthal component and concentrates within a relatively thin cylindrical shell, the Lorentz force can efficiently collimate and confine the jet plasma (see Lupyan and Shukurov, 1992).

As we can see, an application of the theory of the screw dynamo necessitates a detailed knowledge of the velocity field. This fact is the main obstacle in applying the dynamo theory to extragalactic jets. However, we can propose certain qualitative arguments which will clarify whether or not this dynamo mechanism can be of importance in astrophysical jets.

The thermal plasma density in kiloparsec-scale extragalactic jets is estimated from Faraday rotation measurements as $n<10^{-5} \mathrm{~cm}^{-3}$. Then eq. (2) yields the following lower estimate of the shearing velocity required to amplify magnetic field up to a strength of $1 \mu \mathrm{G}$ :

$$
V_{\text {shear }} \simeq 10^{8} \mathrm{~cm} \mathrm{~s}^{-1} \text {. }
$$

Thus, the generation criterion $R_{\mathrm{m}} \simeq 30 V_{\text {shear }} / c_{s}>O(10)$ can be satisfied provided the sound speed $c_{s}$ is less than about a few percent of the speed of light.

Eq. (2) can be used to deduce the variation of the dynamo-generated magnetic field strength along the jet axis. Assuming that the longitudinal velocity (and its shear) varies along $z$ only insignificantly (a supersonic jet), the conservation of mass implies that $\rho \propto R^{-2}$. Therefore, we obtain the relationship $B \propto R^{-1}$ which agrees with the observations of Walker et al. (1987). Within the framework of the present interpretation, this scaling applies to the total regular magnetic field. As the ratio of the longitudinal and transverse components of the magnetic field is controlled by the flow parameters through eq. (1), the field components also follow this scaling separately, provided $\omega$ is also independent of $z$.

Observations of some jets indicate that projected magnetic field orientations exhibit oscillatory behavior along $z$ with the wavelength $k^{-1}=N R$, where $N \approx 5$ for NGC 6251 (Perley et al., 1984) and $N \approx 10$ for 3C 219 (Bridle et al., 1986). This pattern can be attributed to the $m=1$ magnetic mode (Königl and Choudhuri, 1985). Then we obtain from eqs. (1) and (3)

$$
\omega R \simeq v_{z} / N \simeq 10^{7} \mathrm{~cm} \mathrm{~s}^{-1}
$$

if the distributions of $\omega(r)$ and $v_{z}(r)$ have similar radial scales. Königl (1987) argues that this magnetic field configuration can be close to a force-free one resulting from 
magnetic relaxation, which restricts the admissible values of $N$. We stress that the screw dynamo can directly generate such fields, but without any strong restriction on $N$.

The rotation velocity implied by eq. (4) is incompatible with angular momentum conservation even if the matter rotated at the largest possible angular velocity at the inner edge of the accretion disk. Therefore, the origin of rotation of the jet plasma represents a separate physical problem if we wish to apply the above arguments to kiloparsec-scale jets. However, the rotation velocity compatible with angular momentum conservation might be sufficient in parsec-scale jets, so that we can speculate that the screw dynamo can be efficient in some vicinity of the central object generating there magnetic field with the properties described above, which is then advected to the outer parts of the jet.

Our arguments, although tentative, indicate that the screw dynamo can be an important source of magnetic energy in astrophysical jets and this mechanism deserves further attention.

We are grateful for Dr. A.Gilbert for helpful discussion.

\section{References}

Asseo, E.: 1987, in Interstellar Magnetic Fields, ed(s)., R. Beck and R. Gräve, Springer-Verlag: Berlin, 194

Begelman, M.C., Blandford, R.D. and Rees, M.: 1984, Rev. Mod. Phys. 56, 255

Blandford, R.D. and Rees, M.J.: 1974, Mon. Not. Roy. Astron. Soc. 169, 395

Bridle, A.H., Perley, R.A. and Henriksen, R.N.: 1986, Astron. J. 72, 534

Camenzind, M.: 1990, in Reviews of Modern Astronomy, 3, ed(s)., G. Klare, Springer-Verlag: Heidelberg, 234

Camenzind, M. and Krockenberger, M.: 1992, Astron. Astrophys. 255, 59

De Young, D.S.: 1980, Astrophys. J. 241, 81

Gilbert, A.: 1988, Geophys. Astrophys. Fluid Dyn. 44, 241

Gvaramadze, V.V., Lominadze, J.G., Ruzmaikin, A.A., Sokoloff, D.D. and Shukurov, A.M.: 1988, Astrophys. Space Sci. 140, 165

Königl, A.: 1987, in Magnetic Fields and Extragalactic Objects, ed(s)., E. Asseo and D. Grésillon, Editions de Physique: Les Ulis, 271

Königl, A. and Choudhuri, A.R.: 1985, Astrophys. J. 289, 173

Lada, C.J.: 1985, Ann. Rev. Astron. Astrophys. 23, 267

Laing, R.A.: 1981, Astrophys. J. 248, 87

Lortz, D.: 1968, Plasma Phys. 1, 966

Lupyan, E.A. and Shukurov, A.: 1992, Magnit. Gidrodin. 3, 15

Mirabel, I.F., Rodríguez, L.F., Cordier, B., Paul, J. and Lebrun, F.: 1992, Nature 358, 215

Perley, R.A.: 1987, in Magnetic Fields and Extragalactic Objects, ed(s)., E. Asseo and D. Grésillon, Editions de Physique: Les Ulis, 255

Perley, R.A., Bridle, A.H. and Willis, A.G.: 1984, Astrophys. J. Suppl. 54, 291

Ponomarenko, Yu.B.: 1973, Zh. Prikl. Mekh. Tekhn. Fiz. (USSR) 6, 47

Pudritz, R.E.: 1981, Mon. Not. Roy. Astron. Soc. 195, 881, 897

Reshetnyak, M., Sokoloff, D. and Shukurov, A.: 1991, Astron. Nachr. 312,33

Ruzmaikin, A., Sokoloff, D. and Shukurov, A.: 1988, J. Fluid Mech. 197, 39

Ruzmaikin, A.A., Sokoloff, D.D., Solov'ev, A.A. and Shukurov, A.: 1989, Magnetohydrodynamics 25, 6

Sokoloff, D.D., Shukurov, A.M. and Shumkina, T.S.: 1989, Magnetohydrodynamics 25,1

Stepinski, T.F. and Levy, E.H.: 1991, Astrophys. J. 379, 343

Walker, R.C., Benson, J.M. and Unwin, S.C.: 1987, Astrophys. J. 316, 546 